\title{
Artificial lymph nodes induce potent secondary immune responses in naive and immunodeficient mice
}

\author{
Noriaki Okamoto, Risa Chihara, Chiori Shimizu, Sogo Nishimoto, and Takeshi Watanabe \\ Unit for Immune Surveillance Research, Research Center for Allergy and Immunology (RCAI), RIKEN Institute, Yokohama, Japan.
}

\begin{abstract}
We previously demonstrated that artificial lymph nodes (aLNs) could be generated in mice by the implantation of stromal cell-embedded biocompatible scaffolds into their renal subcapsular spaces. $T$ and $B$ cell domains that form in aLNs have immune response functions similar to those of follicles of normal lymphoid tissue. In the present study, we show that the aLNs were transplantable to normal as well as SCID mice, where they efficiently induced secondary immune responses. Antigen-specific secondary responses were strongly induced in aLNs even 4 weeks after their transplantation. The antigen-specific antibody responses in lymphocyte-deficient SCID mice receiving transplanted aLNs were substantial. The cells from the aLNs migrated to the SCID mouse spleen and BM, where they expanded to generate large numbers of antigen-specific antibody-forming cells. Secondary responses were maintained over time after immunization (i.e., antigen challenge), indicating that aLNs can support the development of memory $\mathrm{B}$ cells and long-lived plasma cells. Memory CD4 ${ }^{+} \mathrm{T}$ cells were enriched in the aLNs and spleens of aLN-transplanted SCID mice. Our results indicate that aLNs support strong antigen-specific secondary antibody responses in immunodeficient mice and suggest the possibility of future clinical applications.
\end{abstract}

\section{Introduction}

The host immune response has evolved to resist a wide variety of invading pathogens, and among vertebrates, this process is augmented by the existence of highly organized lymphoid tissues. These structures function at multiple levels. First, they help ensure an encounter between rare antigen-specific $\mathrm{T}$ or $B$ cells and the small number of pathogens that infiltrate the lymph nodes and Peyer's patches, often carried there by DCs or captured in the marginal zone of the spleen. Second, specialized trafficking systems, which are orchestrated by components of the addressin, integrin, and chemokine families, direct the flow of cells into appropriate organs and to sites of inflammation. Lymph nodes are organs formed along the lymphatic vessels and are strategically located to perform immunologic surveillance by capturing free or DC-bound processed foreign antigens and rapidly generating an immune response (1).

The secondary lymphoid tissues are organized into a highly specialized microarchitecture consisting of $\mathrm{B}$ and $\mathrm{T}$ cell domains that promote the interaction of lymphocytes with cognate antigen as well as the collaboration of $\mathrm{B}$ and $\mathrm{T}$ cells to generate $\mathrm{T}$ cell-dependent antibody responses. The $\mathrm{B}$ cell domain, or follicle, is the site where with $T$ cell help, antigen-stimulated B cells multiply and form germinal centers. A special cell type, the follicular DC (FDC), extends projections into the center of a follicle and forms a network structure. The vascular system in the secondary lymphoid tissues also contrib-

Nonstandard abbreviations used: AFC, antibody-forming cell; aLN, artificial lymph node; CCR, CC chemokine receptor; CD62L, CD62 ligand; CDR, complementarity determining region; CXCR, CXC chemokine receptor; CX3CR1, CX3C chemokine receptor 1; EGFP, enhanced green fluorescent protein; ELISPOT, enzyme-linked immunospot assay; FDC, follicular DC; LT $\alpha$, lymphotoxin $\alpha$; NIP, (4-hydroxy-3-iodo5-nitrophenyl) acetyl; NP, (4-hydroxy-3-nitrophenyl) acetyl; PTX, pertussis toxin; S1P, sphingosine-1-phosphate.

Conflict of interest: The authors have declared that no conflict of interest exists. Citation for this article: J. Clin. Invest. 117:997-1007 (2007). doi:10.1172/JCI30379. utes to immune surveillance. In particular, there are high endothelial venules through which lymphocytes enter the lymph nodes (2).

Studies using gene targeting and spontaneous mouse mutants have identified factors important for the development of secondary lymphoid tissues and maintenance of the normal microarchitecture. This was first shown for the TNF family member lymphotoxin $\alpha$ (LT $\alpha)$. There were no Peyer's patches in the LT $\alpha$ knockout mouse and greatly decreased numbers of lymph nodes $(3,4)$. The lymphotoxin $\beta$ receptor-deficient (LT $\beta$ R-deficient) mouse has a more severe phenotype, with a complete loss of lymph nodes throughout the body (5-7). The alymphoplasia (aly) mouse mutation results in a complete loss of Peyer's patches and lymph nodes. The aly defect is caused by a point mutation in NF-KB-inducing kinase, a signaling molecule downstream of the LT $\beta$ R (8). Because normal immune responses do not occur in mice with no lymph nodes, the aly mice are severely immunodeficient.

We previously demonstrated that artificial lymph node-like (aLN-like) tissues could be generated by implanting stromal cell-embedded biocompatible scaffolds into the renal subcapsular spaces of mice. These aLNs possess a well-organized tissue structure similar to that of the secondary lymphoid organs (9). As in natural lymph nodes, there are T cells, B cells, and DCs; T and $\mathrm{B}$ cell domains are clearly distinguishable; and follicles develop. Germinal centers develop in the aLNs following immunization, an important feature of a secondary lymphoid organ, and there is vigorous $\mathrm{B}$ cell proliferation and plasma cell generation. T and $\mathrm{B}$ cell domains that form in the aLNs are not simply nonspecific cellular aggregates, but function in the immune response in a manner similar to the follicles of normal lymphoid tissue. The aLNs are transplantable to naive as well as SCID mice and efficiently induce secondary immune responses.

Here we report the further analyses of immune functions mediated by the aLNs. The aLNs supported extremely potent antigenspecific secondary antibody responses in SCID mice. Cells in the 

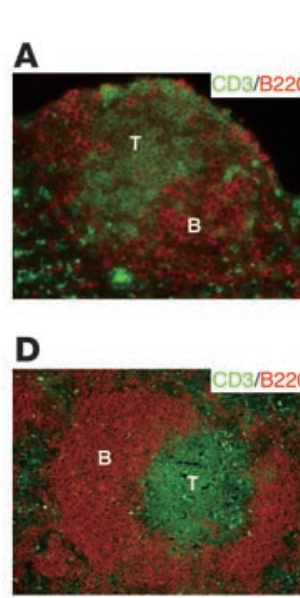

B

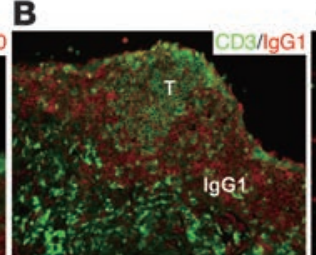

ค'
aLN

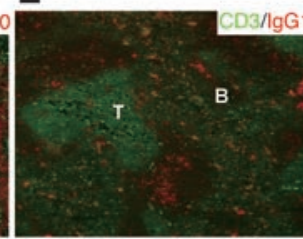

c

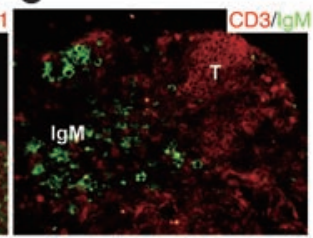

$\mathbf{F}$

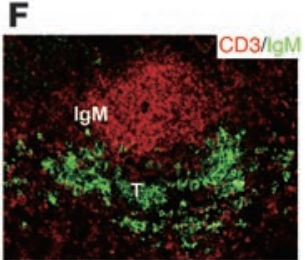

G

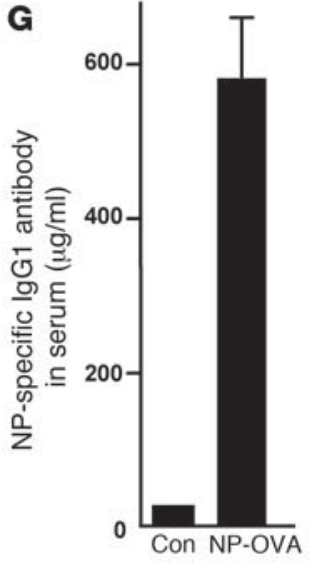

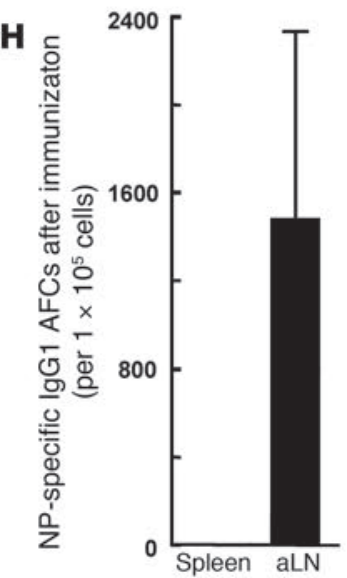

Figure 1

Antigen-specific secondary lgG responses are induced in transplanted aLNs, but not in the spleens, of normal naive recipients even 4 weeks after transplantation. The aLNs were formed first in NP-OVA-preimmunized BALB/c mice. After their formation, aLNs were removed and transplanted into renal subcapsular regions of naive, nonimmunized BALB/c mice. Mice were kept in cages for 3 weeks and then immunized i.v. with NP-OVA antigen (100 $\mu \mathrm{g} / \mathrm{mouse})$. Tissue sections of aLNs and sera of the recipient mice were collected 5 days after immunization. (A-F) Immunohistochemical staining of aLNs $(\mathbf{A}-\mathbf{C})$ and spleens of aLN-transplanted mice (D-F) 4 weeks after transplantation. Antibodies used were as follows: FITC-labeled anti-mouse CD3, biotin-labeled anti-mouse B220, and Qdot 605 streptavidin conjugate (A and D); FITC-labeled antimouse CD3, biotin-labeled anti-mouse IgG1, and Qdot 605-labeled streptavidin (B and E); FITC-labeled anti-mouse IgM, biotin-labeled antimouse CD3, and Qdot 605 streptavidin conjugate (C and F). T, T cell; B, B cell; IgG1, IgG1 AFC; IgM, IgM AFC. Original magnification, $\times 100$. (G) IgG1 NP-specific antibody titers in sera of aLN-transplanted BALB/c mice without immunization (Con) or immunized with NP-OVA. (H) Number of IgG1 NP-specific AFCs in aLNs and spleens of recipient BALB/c mice after immunization with NP-OVA.

transplanted aLNs also migrated to the SCID mouse spleen and $\mathrm{BM}$, where they expanded to generate large numbers of antigenspecific antibody-forming cells (AFCs). Our results indicated that movement of cells from the aLNs to the spleen is mediated through signaling from pertussis toxin-sensitive (PTX-sensitive) $G$ protein-coupled receptors, including chemokine receptors and sphingosine-1-phosphate (S1P) receptors. The number of antigen-specific AFCs was maintained over time after immunization (i.e., antigen challenge), indicating that aLNs can support development of memory B cells and long-lived plasma cells. Memorytype $\mathrm{CD}^{+} \mathrm{T}$ cells were enriched in the aLNs and in spleens of recipient SCID mice. T cells positive for CD127 (also referred to as IL-7 receptor $\alpha$ ) were also enriched in the aLNs. During the initial stages of aLN formation, a massive accumulation of FDC-M1positive, FDC-like cells was observed. The appearance of these FDC-like cells preceded the formation of $\mathrm{T}$ cell areas and $\mathrm{B}$ cell follicles and thus may be involved in establishing the groundwork for the lymph node microarchitecture.

\section{Results}

Effective induction of secondary antibody response in aLN-transplanted naive mice. Cells from the stromal cell line TEL-2-LT $\alpha(9,10)$ as well as BM-derived mature DCs (11) embedded in scaffolds were implanted into the renal subcapsular spaces of BALB/c mice preimmunized with alum-precipitated (4-hydroxy-3-nitrophenyl) acetyl-OVA (NP-OVA). Three weeks after implantation, aLNs had formed in the recipient mice, as reported previously (9). These newly formed aLNs were then removed and transplanted into the renal subcapsular spaces of naive BALB/c mice. After 3-4 weeks, the recipient mice were immunized by i.v. injection of NP-OVA (100 $\mu \mathrm{g} /$ mouse), and 5 days after immunization, the histology of the transferred aLNs was examined by immunofluorescence staining.
The structure of aLNs was intact, with clearly segregated T and B cell areas, in naive recipient mice even 4 weeks after transplantation (Figure 1A). Large numbers of IgG1 AFCs, but few IgM AFCs, were detected in B cell areas of the transplanted aLNs (Figure 1, B and C), whereas the inverse was observed in spleens of the recipient mice (Figure 1, D-F). At this time point, a large amount of IgG1 NP-specific antibody $(500-800 \mu \mathrm{g} / \mathrm{ml})$ was detected in the sera of recipient mice (Figure 1, G and $\mathrm{H}$ ). These data indicate that the tertiary structure of the aLNs is stable and persistent even 4 weeks after transplantation and that a potent antigen-specific IgG1 secondary antibody response could be induced specifically within aLNs.

We next performed transplantation experiments using aLNs formed in enhanced green fluorescent protein (EGFP) transgenic mice, which allowed the unambiguous identification of donor and recipient cells. The aLNs were generated in the renal subcapsular spaces of EGFP transgenic mice by the implantation of collagen sponges containing stromal cells, TEL-2-LT $\alpha$ cells, and DCs generated from normal BALB/c BM cells. Most of the immune cells in the aLNs were found to be host derived and EGFP positive (Figure 2, A-F). Both $\mathrm{T}$ and $\mathrm{B}$ cell areas were formed by EGFP-positive cells (Figure 2, A and D-F), and EGFP-positive DCs were scattered in both $\mathrm{T}$ and $\mathrm{B}$ cell areas (Figure 2C). FDC networks were detected in the $\mathrm{B}$ cell area, all of which appeared to be EGFP positive (Figure 2B). These data indicate that most of the immune cells that comprise the aLNs are derived from the recipient mouse.

The kinetics of aLN formation were next examined. There was a massive influx of FDCs in scaffolds 3 days after implantation of the TEL-2 stromal cell-embedded collagen sponge into renal subcapsular spaces in BALB/c mice (Figure 3, B and F) prior to the infiltration of any host lymphocytes (Figure 3, A and E), and this was followed by formation of distinct $\mathrm{T}$ and $\mathrm{B}$ cell areas (Figure 3, B-D and $\mathrm{F}-\mathrm{H})$. Some FDC-M1-positive cells were initially present in $\mathrm{T}$ 


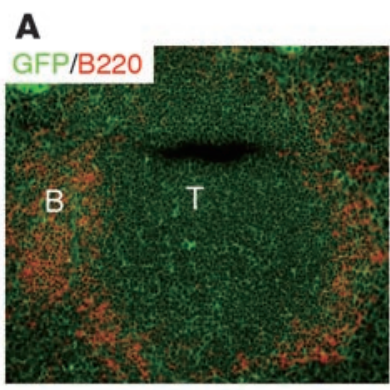

B

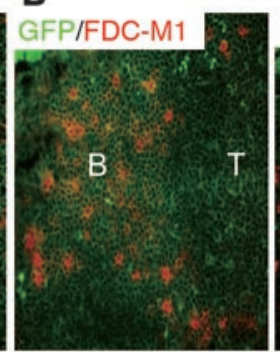

E

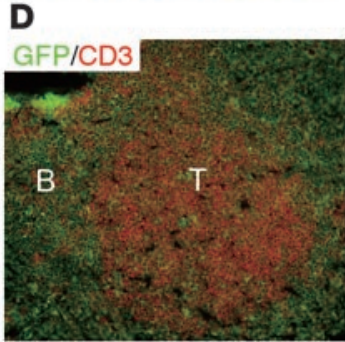

G

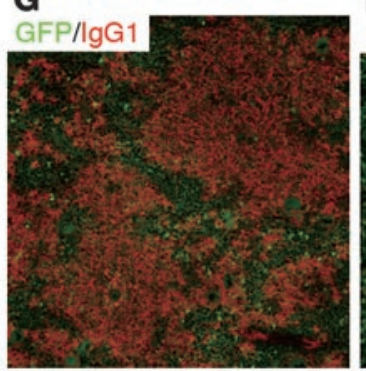

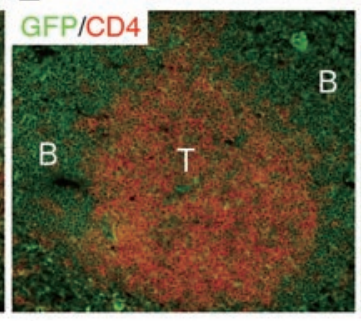

H

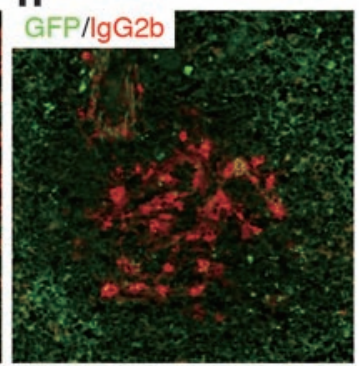

C

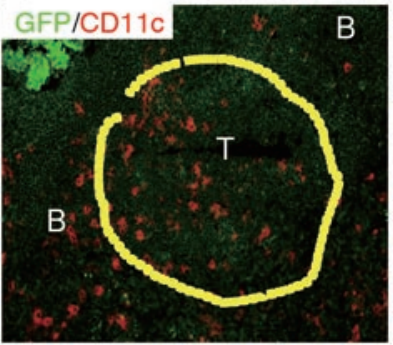

$\mathbf{F}$

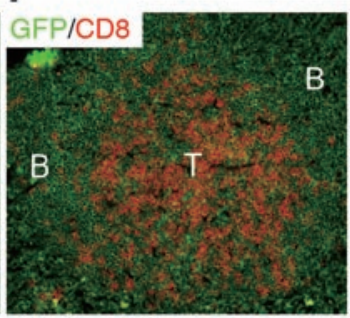

B

\section{Figure 2}

Generation of aLNs. Immunohistochemical staining shows that aLNs formed in NP-OVA-preimmunized EGFP transgenic BALB/c mice (A-F). After their formation, aLNs were removed and transplanted into renal subcapsular regions of SCID mice, and then mice were immunized intravenously with NP-OVA. Immunohistochemical staining in the spleens of SCID mouse recipients carrying aLNs $(\mathbf{G}$ and $\mathbf{H})$. Spleen cells were examined after the second immunization with NP-OVA. Increased numbers of IgG1 AFCs (G), compared with few IgG2 AFCs $(\mathbf{H})$, were observed in SCID mouse spleens. Antibodies used were as follows: biotin-labeled anti-mouse B220 and Qdot 605 streptavidin conjugate (A); anti-mouse FDC-M1 and Alexa Fluor 594 conjugate (B); biotin-labeled antimouse CD11c and Qdot 605 streptavidin conjugate (C); biotin-labeled anti-mouse CD3 and Qdot 605 streptavidin conjugate; biotin-labeled anti-mouse CD4 and Qdot 605 streptavidin conjugate (E); biotinlabeled anti-mouse CD8 and Qdot 605 streptavidin conjugate (F); biotin-labeled anti-mouse IgG1 and Qdot 605 streptavidin conjugate (G); biotin-labeled anti-mouse IgG2b and Qdot 605 streptavidin conjugate (H). (C) The area circled in yellow shows the $\mathrm{T}$ cell area. Original magnification, $\times 100$. cell areas, but these became strictly localized in the $\mathrm{B}$ cell region (Figure 3, D and H). These observations strongly suggest that the appearance of FDC-like cells at an early stage of the aLN formation may play a crucial role in the formation of not only B cell follicles but also the T cell region in aLNs. Staining of small vessels and capillaries with tomato lectin showed that the aLNs were highly vascularized, with blood vessels connected to the major blood vessels in the kidney capsules (Supplemental Figure 1; supplemental material available online with this article; doi:10.1172/JCI30379DS1).

Induction of strong secondary antibody responses in immunodeficient hosts with aLNs. To examine whether aLNs restore immune function in immunodeficient hosts, we performed transplantation experiments using SCID mice as recipients. aLNs generated in NP-OVApreimmunized $\mathrm{BALB} / \mathrm{c}$ mice were removed and transplanted into the renal subcapsular spaces of SCID mice (assigned as day 0 ). On day $1,100 \mu \mathrm{g}$ NP-OVA was administered i.v. to the recipient mice, and on day 7, the mice were boosted i.v. with $10 \mu \mathrm{g}$ NP-OVA. Highand low-affinity IgG1 NP-specific AFCs were detected (with $\mathrm{NP}_{3}$ OVA and $\mathrm{NP}_{30}$-OVA, respectively, as detecting antigens) by enzymelinked immunospot assay (ELISPOT) in the transferred aLNs as well as spleens and BM of the recipient mice 5 days after the second immunization. As shown in Figure 4A, many high-affinity IgG1 NP-specific AFCs were detected in the aLNs after both NP-OVA injections. At this time point, a substantial amount of high-affinity IgG1 NP-specific antibody $(7,258.9 \pm 707.9 \mu \mathrm{g} / \mathrm{ml})$ was found in the sera of recipient mice (Table 1 ). Since the amount of serum NP-specific antibodies produced by aLNs in the recipient naive mice was at most about $500-800 \mu \mathrm{g} / \mathrm{ml}$ (Figure 1G), these large amounts of
NP-specific antibodies could not be explained solely by their production from aLNs. To clarify the origin of these antibodies, the number of IgG1 NP-specific AFCs was determined in the spleens and BM of the recipient SCID mice by ELISPOT. A large number of IgG1 NP-specific AFCs were detected in the spleens of aLN-transplanted SCID mice after the second i.v. injection of NP-OVA (Table 2). Amazingly, one-third of the splenic lymphocytes in these mice were high-affinity IgG1 class NP-specific AFCs. In addition, the number of IgG1 NP-specific AFCs increased over time both in the aLNs and in the spleens of the recipient SCID mice over 2 weeks after the second immunization, comprising almost half the spleen cells (Figure 4, A and B). Similarly, a large number of NP-specific AFCs was detected in BM cells of the recipient mice (Figure 4C). Only a small number of IgG1 NP-specific AFCs were detected in the spleens of aLN-transplanted SCID mice after the first injection of NP-OVA, but few were detected in spleens after the first or second injection of NP-OVA in naive aLN-transplanted mice (Figure 4D). We also examined the effect of varying the time of the first immunization after transplantation on subsequent antibody responses. NP-OVA was administered i.v. to the SCID mice on the seventh day after aLN transplantation. Regardless of the time of first immunization, the increase in AFCs after the second immunization showed the same behavior in aLNs and spleens (Figure 4, E and F).

We next performed similar transplantation experiments using aLNs formed in NP-OVA-preimmunized EGFP transgenic mice and SCID mice as recipients. Following transplantation, NP-OVA was administered i.v. to the recipient mice 2 times with a 1 -week interval. Large numbers of EGFP-positive cells were detected in 

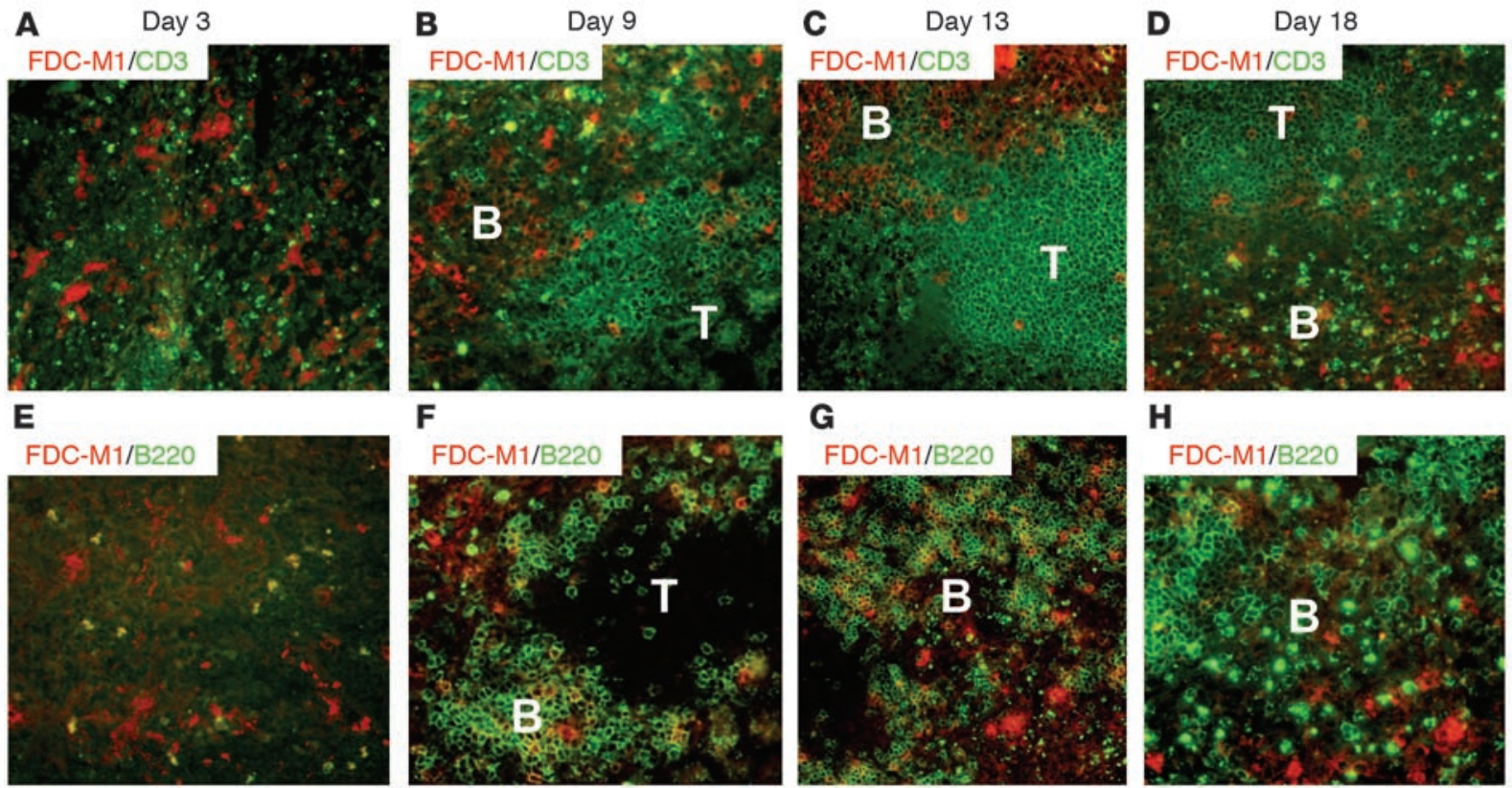

Figure 3

Kinetics of aLN formation. Immunohistochemical staining of the cells in TEL-2-LT $\alpha$ stromal cell-embedded collagen sponges in BALB/c mice. The time course of aLN formation was examined 3, 9, 13, and 18 days after inoculation of collagen sponge into renal subcapsular space in $\mathrm{BALB} / \mathrm{c}$ mice. Antibodies were as follows: FITC-labeled anti-mouse CD3, anti-mouse FDC-M1, and Alexa Fluor 594-conjugated anti-rat IgG (A-D); FITC-labeled anti-mouse B220, anti-mouse FDC-M1, and Alexa Fluor 594-conjugated anti-rat IgG (E-H).

the spleens of SCID mice after the second immunization. In the SCID mouse spleens, most of the EGFP-positive B cells were IgG1 positive, but a few IgG2-producing cells were found in the B cell areas (Figure 2, G and $\mathrm{H}$ ). On the other hand, only small numbers of EGFP-positive cells were detected in the spleens of aLN-transplanted SCID mice after the first injection of NP-OVA. The EGFPpositive IgG1-producing cells rapidly and explosively appeared in spleens and BM shortly after the second i.v. injection of NP-OVA. Taken together, these results indicate that most of the AFCs in aLNs remained within these structures when they were transplanted into naive normal recipients. However, when the aLNs were transplanted into SCID mice, which have lymphocyte-free immune tissues, the $B$ and $T$ cells migrated from the aLNs into their spleens and $\mathrm{BM}$, expanded explosively, and rapidly matured to become antigenspecific IgG class antibody-secreting plasma cells.

$V_{H}$ gene diversity of the NP-specific antibodies produced by aLNs transplanted into SCID mice. Since large numbers of NP-specific AFCs were obtained by transfer of aLNs into SCID mice followed by i.v. immunization, antigen-specific hybridomas could be easily established by fusion between plasmacytoma cells and aLN-derived cells or spleen cells of aLN-transplanted SCID mice. Almost all of the spleen cell-derived hybridomas were found to produce high-affinity IgG1 NP-specific antibodies (data not shown). The $V_{H}$ genes used by these IgG1 NP-specific antibodies were determined for 146 of the hybridomas. The IgG1 antibodies were classified into 8 groups by comparing their D-J regions (Table 3 ). About $70 \%$ of the sequences fell into group 1 . There were few differences between complementary determining region 1 (CDR1) and CDR2, and no differences in $C D R 3$, when the $V_{H}$ gene sequences within group 1 were compared (Figure 5), suggesting that the diversity of the high-affinity NP-specific antibodies produced by aLNs was highly restricted and oligoclonal. The $\kappa$ light chain was used by all investigated hybridomas (data not shown). These data indicate that vigorous somatic hypermutation and affinity maturation in the single B cell clone specific for NP-hapten could be induced in aLNs or aLN-derived $\mathrm{B}$ cells in SCID mouse spleen.

Accumulation of memory-type CD4 $4^{+}$T cells and memory $B$ cells in aLNs. TEL-2-LT $\alpha$ cells and activated DCs embedded in scaffolds were transplanted into BALB/c mice that had been preimmunized with alum-precipitated NP-OVA. Three weeks after the transplantation, the aLNs and endogenous recipient mouse lymph nodes were collected and examined by FACS or immunohistochemistry. No difference in the ratio of $\mathrm{CD}^{+}$to $\mathrm{CD}^{+} \mathrm{T}$ cells was observed between aLNs and recipient lymph nodes. Surprisingly, however, the population of CD44-high, CD62 ligand-low (CD44hi CD62L ${ }^{\text {lo }}$ ) memory-type $\mathrm{CD} 4^{+} \mathrm{T}$ cells was enriched in aLNs (Figure 6A). In aLNs, $80.1 \%$ of $\mathrm{CD}^{+}{ }^{+} \mathrm{T}$ cells were of the CD44hiCD62 $\mathrm{L}^{\text {lo }}$ memory type, compared with $16.5 \%$ in the host lymph nodes. In other experiments, aLNs

\section{Table 1}

lgG1 NP-specific antibody in serum
aLN-transplanted SCID (nonimmunized)

aLN-transplanted SCID

(immunized twice)

SCID (immunized twice)

Preimmunized BALB/C

(immunized twice)
IgG1 NP-specific antibody $(\mu \mathrm{g} / \mathrm{ml})$ $0.61 \pm 0.32$

$7,258.9 \pm 707.9$

$2.8 \pm 0.0$

$673.3 \pm 271.8$
Values are mean \pm SEM 
A
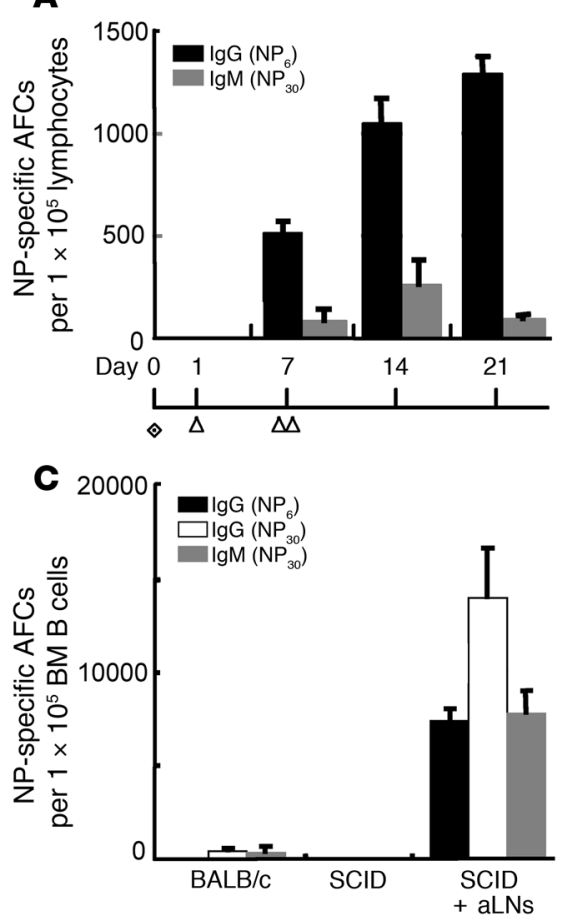

E

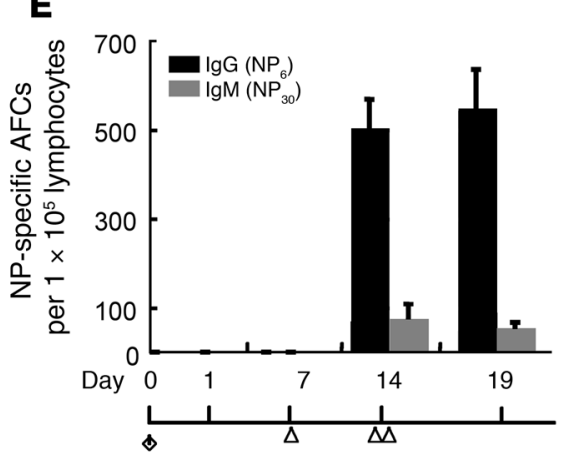

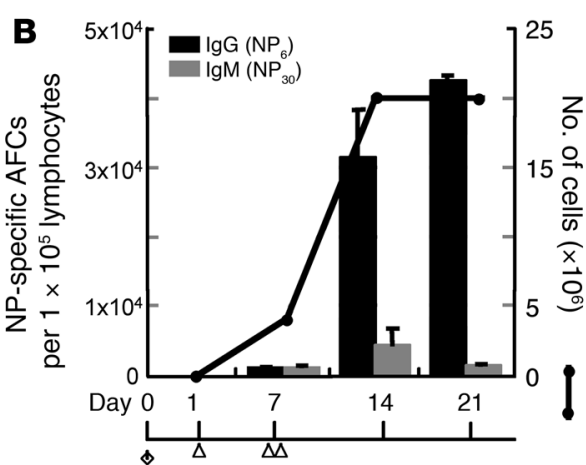
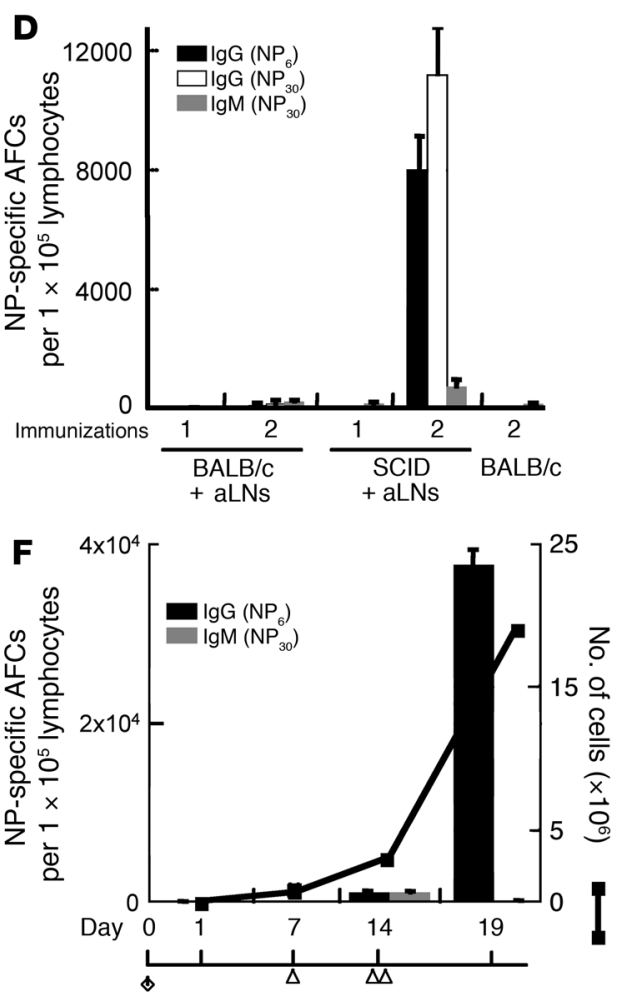

Figure 4

Increased number of IgG1 NP-specific AFCs in aLN-transplanted SCID mice after immunization with NP-OVA. (A and B) Number of NP-specific IgG AFCs in aLNs (A) or spleens (B) of aLN-transplanted SCID mice. Mice were immunized i.v. with NP-OVA on days 1 and 7 (100 $\mu \mathrm{g} / \mathrm{mouse}$ and $10 \mu \mathrm{g} / \mathrm{mouse}$, respectively) after transplantation of aLNs into SCID mice on day 0 . The number of lymphocytes in spleens of aLN-transplanted SCID mice before and after NP-OVA immunization is denoted by the line (B). (C) Upon the second antigen stimulation, large numbers of IgG1 NP-specific AFCs were also detected in the BM of aLNtransplanted SCID mice. (D) Number of NP-specific IgG AFCs in spleens of recipient SCID and BALB/c mice. (E and F) Number of NP-specific IgG AFCs in aLNs (E) or spleens (F) of aLN-transplanted SCID mice immunized i.v. with NP-OVA on days 7 and $14(100 \mu \mathrm{g} /$ mouse and 10 $\mu \mathrm{g} /$ mouse, respectively) after transplantation of aLNs into SCID mice on day 0 . The number of lymphocytes in spleens of aLN-transplanted SCID mice before and after NP-OVA immunization is denoted by the line $(\mathbf{F})$. formed in $\mathrm{BALB} / \mathrm{c}$ mice were transplanted into SCID mice, and then NP-OVA was administered twice i.v. The number of CD $44^{\text {hi }} \mathrm{CD} 62 \mathrm{~L}^{\text {lo }}$ memory-type $\mathrm{CD}^{+} \mathrm{T}$ cells was also enriched, up to $60 \%$ in spleens of aLN-transplanted SCID mice (Figure $6 \mathrm{~B}$ ). $\mathrm{CD} 127^{+} \mathrm{T}$ cells were barely detectable in the lymph nodes of aLN-transplanted BALB/c mice, but were highly enriched in aLNs (Figure 6C).

Accumulation of germinal center $B$ cells and memory $B$ cells in the aLN-derived spleen cells of SCID mice. The B cell profile in spleens of aLN-transplanted SCID mice showed a large number of germinal center $\mathrm{B}$ cells $\left(\mathrm{CD} 38^{\mathrm{lo}} \mathrm{IgG} 1^{+}\right)$and an enrichment of memory B cells $\left(\mathrm{CD} 38^{\mathrm{hi}} \mathrm{IgG} 1^{+}\right)$ as well as a large number of IgG NP-specific AFCs $\left[\mathrm{IgG} 1^{+}\right.$and (4-hydroxy-3-iodo-5-nitrophenyl) acetyl-BSA-positive; NIP-BSA $\left.{ }^{+}\right]$after the second antigen stimulation (Figure 7).

Lymphocyte migration from aLNs into SCID recipient lymphoid tissues. Various receptors transduce signals in immune cells by associating with $G$ proteins, and the signals modulate cellular motility (e.g., S1P and chemokine receptors; refs. 12, 13). PTX from
Table 2

IgG1 NP-specific AFCs in aLN-transplanted SCID mice

Bordetella pertussis is an ADP-ribosylating toxin that prevents G protein-coupled receptor activation and consequently disrupts the signal transduction cascade for lymphocyte egress $(14,15)$. In order to clarify whether $\mathrm{G}$ protein-coupled receptors are involved in the migration of lymphocytes from aLNs to peripheral lymphoid tissues in SCID mice, aLNs were produced in BALB/c mice that had been immunized with alum-precipitated NP-OVA. The aLNs were then cultured in vitro for 3 hours in medium contain- 


\section{Table 3}

Classification of $V_{H}$ genes of Ig $G 1$ antibodies based on $V, D$, and $J$ gene segment usage

\begin{tabular}{ccccc} 
Group & V & D & J & $\begin{array}{c}\text { No. of clones } \\
\text { (\% of total clones) }\end{array}$ \\
1 & IGHV1S128 (J558c) & ST4 & $J_{H} 2$ & $104(71)$ \\
2 & IGHV1S133 (J558i) & FL16.1 & $J_{H} 2$ & $14(10)$ \\
3 & IGHV1S128 (J588C) & FL16.1 & $J_{H} 1$ & $6(4)$ \\
4 & IGHV1-7 (IGHV1S26,H30) & Q52 & $J_{H} 3$ & $6(4)$ \\
5 & IGHV5S16 (98-3G) & SP2.13 & $J_{H} 4$ & $2(1)$ \\
6 & IGHV1-67 (IGHV1S137,J558n) & FL16.1 & $J_{H} 4$ & $4(3)$ \\
7 & IGHV1-48 (IGHV1S8,V108A) & SP2.9 & $J_{H} 4$ & $2(1)$ \\
8 & IGHV1-48 (IGHV1S8,V108A) & SP2.2 & $J_{H} 4$ & $8(5)$ \\
\hline
\end{tabular}

A total of 146 clones were counted.

ing PTX before transplantation into SCID mice. After 2 i.v. immunizations with NP-OVA as described above, aLNs, spleens, and $\mathrm{BM}$ cells of recipient mice were collected. The number of IgG1 NP-specific AFCs in aLNs was greatly increased by treatment with PTX before transfer into SCID mice (Figure 8A). Conversely, the number of IgG1 NP-specific AFCs in spleens or BM of recipient mice was decreased (Figure 8, B and C). As an independent experimental approach, we used the immunosuppressant FTY720. The phosphorylated form of FTY720 binds to 4 of the 5 known S1P receptors on target cells $(16,17)$, which results in a marked downregulation of the receptors and inhibition of lymphocyte emigration from secondary lymphoid tissues (18). We transplanted aLNs generated in $\mathrm{BALB} / \mathrm{c}$ mice that were preimmunized with alum-precipitated NP-OVA into SCID mice, and NP-OVA was then administered i.v. twice with a 1-week interval. FTY720 was administered 3 times after the first NP-OVA immunization. The number of IgG1 NP-specific AFCs in aLNs increased when FTY720 was administered 3 times after the first immunization (Figure $8 \mathrm{D}$ ), suggesting that S1P receptors are important for lymphocyte migration from aLNs into the lymphoid tissues of the SCID mice. We also examined expression of several chemokine receptors in the presence or absence of the initial NP-OVA immunization by FACS analysis. After the first immunization, expression of CC chemokine receptor 7 (CCR7) and CXC chemokine receptor 5 (CXCR5) decreased in the $\mathrm{T}$ and $\mathrm{B}$ cells in the aLNs of SCID recipient mice, but not in those of aLNs of normal BALB $/ c$ recipient mice (Figure $9 \mathrm{~A}$ ). We also examined expression of chemokine receptors by a quantitative RT-PCR method. The expression of CCR5, CXCR6, and CX3C chemokine receptor 1 (CX3CR1) in cells of aLNs of SCID recipient mice was slightly but significantly decreased after NP-OVA immunization, while the expression of the same receptors was slightly increased after immunization in aLNs of naive BALB/c recipient mice (Figure 9B). By contrast, expression of the S1P receptor was unchanged following antigen stimulation. Taken together, these results indicate that signals from PTX-sensitive G protein-coupled receptors, including chemokine receptors and S1P receptors, play a pivotal role in the regulation of lymphocyte migration from the aLNs to the spleens and BM of SCID mice.

\section{Discussion}

We have previously demonstrated that transplantation into the renal subcapsular spaces of mice of a thymus-derived stromal cell line embedded along with DCs in a biocompatible scaffold can efficiently generate lymphoid tissue-like organoids (e.g., aLNs). Structures resembling high endothelial venules, which are crucial for cellular migration in secondary lymphoid organs, have also been observed in the aLN (9), and we recently detected the formation of lymph vessels. The normal generation of lymph nodes requires the passage of various cell types through the blood and lymphatic vasculature into the developing organ. Vascular growth is also important in the generation of aLNs, and the initial vascular growth in the aLN appears to be associated with the thymus-derived stromal cell line embedded along with DCs into the biocompatible scaffold $(9,19)$ (Supplemental Figure 1).

It is a common finding that tissues that harbor the target antigen of chronic immune responses are infiltrated by cellular effectors of the immune system, mainly $\mathrm{T}$ cells and macrophages, but also DCs, B cells, and plasma cells. Intriguingly, it has been observed that these cellular elements can organize themselves microanatomically, as do secondary lymphoid organs, leading to the de novo formation of $\mathrm{B}$ cell follicles and $\mathrm{T}$ cell areas, a phenomenon referred to as lymphoid neogenesis or tertiary lymphoid organ formation (20-26). Tertiary lymphoid organs resolve completely after therapeutic treatment (27), but the aLNs did not resolve in vivo for a long time and retained antigen-specific AFCs even several weeks after antigen stimulation. It thus appears that an aLN is functionally different from a tertiary lymphoid organ.

aLNs generated in preimmunized recipient mice promoted the production of large numbers of antigen-specific high-affinity IgG1 AFCs. The aLN structure and antibody-producing capacity were maintained even after transfer into naive or SCID mice for more than 2 weeks after immunization. We confirmed that antigen-specific high-affinity IgG1 AFCs were found in abundance in spleens and BM of aLN-transplanted SCID recipient mice. We also examined the mechanisms of lymphocyte migration from an aLN to the spleen. It has previously been reported that S1P is a biologically active lysophospholipid that transmits signals through a family of $G$ protein-coupled receptors to control the trafficking of lymphocytes in secondary lymphoid organs and the migration of B cells into splenic follicles $(28,29)$. Our data suggest that a signal from a S1P receptor and decreased expression of chemokine receptors participate in the lymphocyte migration from aLNs to spleen we observed in SCID mice. In addition, our data suggest that antigen stimulation is the trigger for lymphocyte migration from aLNs. Several chemokine receptors on $\mathrm{T}$ cells showed a slight but significant decrease in expression upon antigen stimulation: CCR7 and CXCR5 (assessed by FACS), which are expressed on naive $\mathrm{T}$ cells and $\mathrm{B}$ cells, respectively, and CCR5, CXCR6, and CX3CR1 (assessed by quantitative PCR), which are expressed on Th1 cells, memory $\mathrm{T}$ cells, and $\mathrm{CD}^{+} \mathrm{T}$ cells, respectively. On the other hand, expression of those chemokine receptors was unchanged or upregulated in cells from aLNs transplanted into normal mice, in which no migration of aLN cells to host lymphoid organs occurred. These changes in chemokine receptor expression are likely involved in regulating the different patterns of lymphocyte migration from aLNs into SCID lymphoid tissues, although the precise mechanism is as yet unknown. We propose that the small numbers of $T$ and B cells (IgG1 class-switched memory $\mathrm{B}$ cells) that migrated from the aLNs to the spleens and BM after the first immunization undergo a massive clonal expansion with a restricted $V_{H}$ gene diversity upon the second immunization in SCID mice, which possess large empty niches for lymphocyte proliferation. 

G.

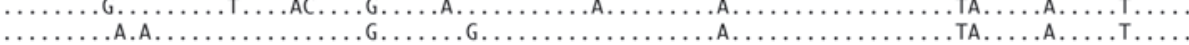

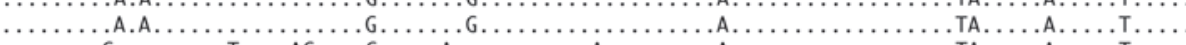

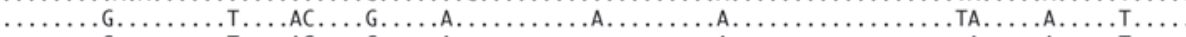

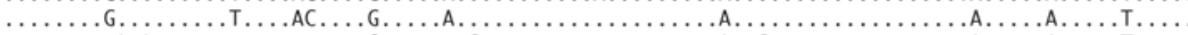

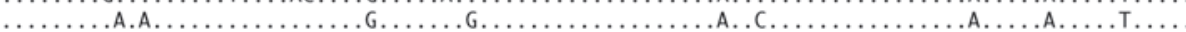

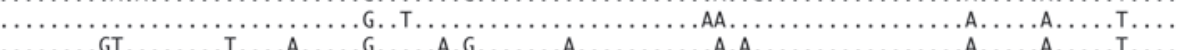

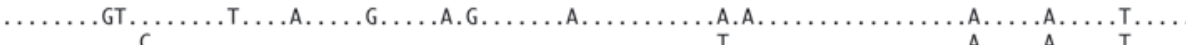

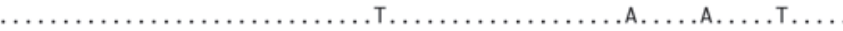

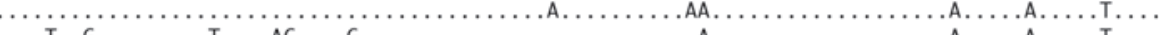

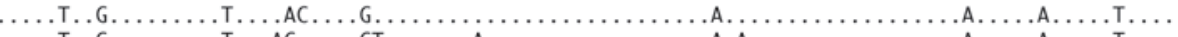

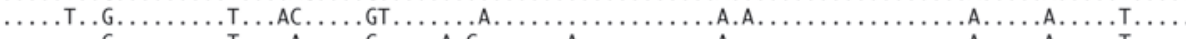

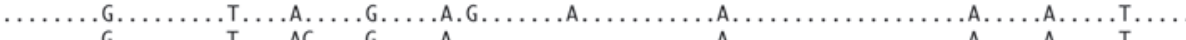

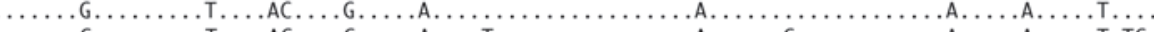

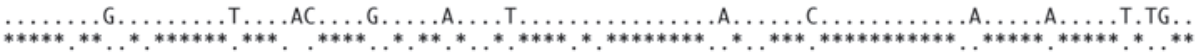

96 CTGGATGCACTGGGTGAAGCAGAGGCCTGGACAAGGCCTTGAGTGGATTGGAGAGATTAATCCTAGCAACGGTCGTACTAACTACAATGAGAAGT

1 C3-1

7B3-1

T9-1

T10-1

1C4-1

1C1-2

$1 \mathrm{~B} 4-1$

2A4-2

3A3-

7A2-2

7B1-2

$7 \mathrm{~B} 2-2$

$7 C 2-1$

7C2-3

8A2-1

$5 \mathrm{~B} 4-2$

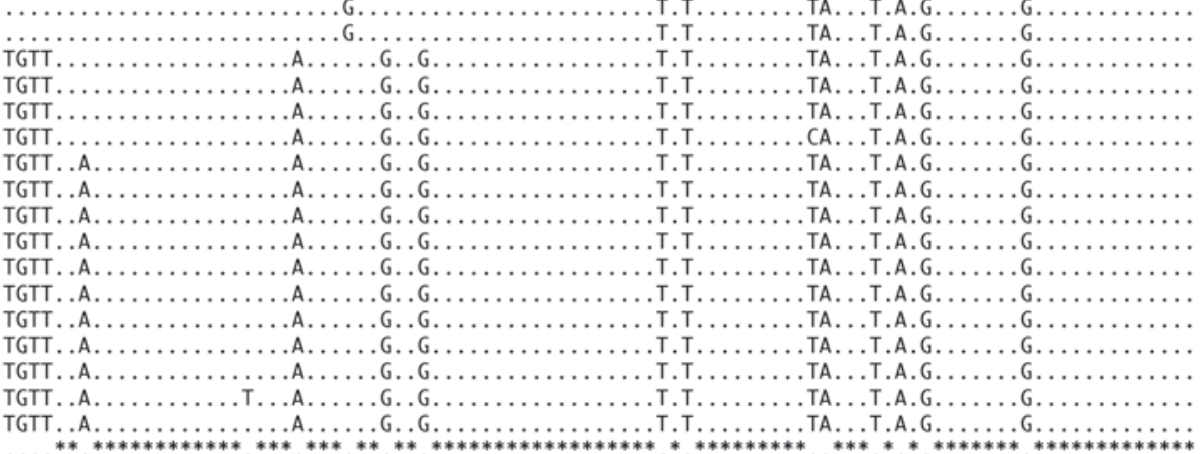

191

BALB/c TCAAGAGCAAGGCCACACTGACTGTAGACAAATCCTCCAGCACAGCCTACATGCAACTCAGCAGCCCGACATCTGAGGACTCTGCGGTCT

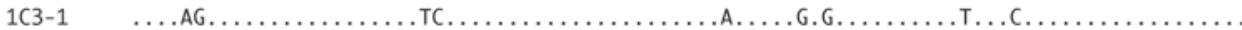

$7 \mathrm{~B} 3$

T9-1

$\mathrm{T} 10-1$

1C4-1

1C1-2

1B4-1

2A4-

$3 A 3-2$

7A2-2

7B1-2

$7 \mathrm{~B} 2-2$

$7 \mathrm{C} 2-1$

7C2-3

8A2-1

5B4-2

$8 \mathrm{C} 4-1$
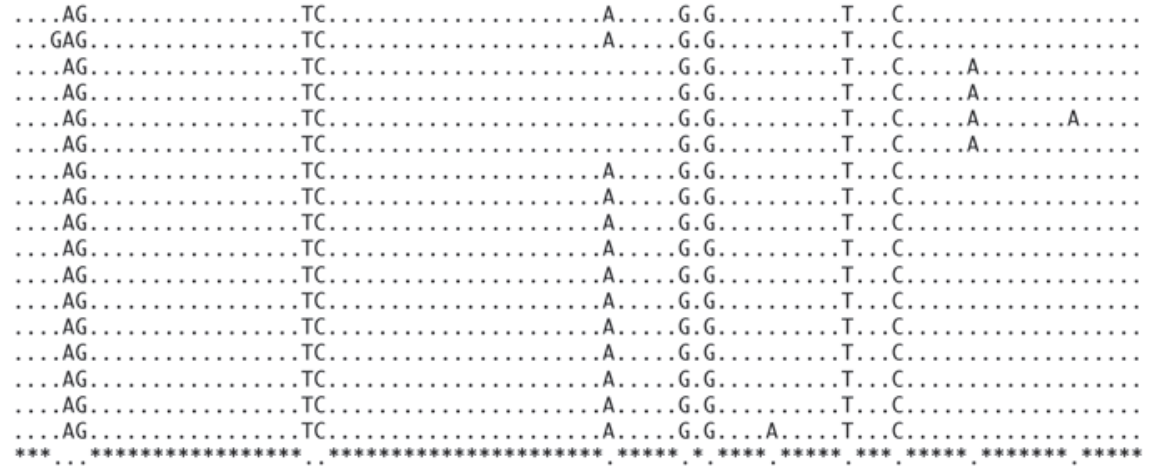

281 330

1C3-1
$7 B 3-1$
T9-1
T10-1
$1 C 4-1$
$1 C 1-2$
$1 B 4-1$
$2 A 4-2$
$3 A 3-2$
$7 A 2-2$
$7 B 1-2$
$7 B 2-2$
$7 C 2-1$
$7 C 2-3$
$8 A 2-1$
$5 B 4-2$
$8 C 4-1$

ATTACTGTGCAAGAAGGCAGCTCGGGCTACCCCCGTACTACTTTGACTAC

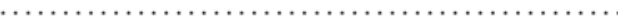

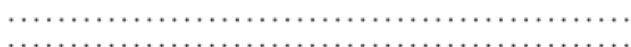

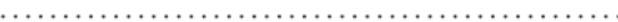

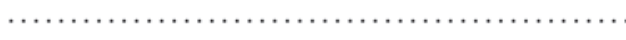

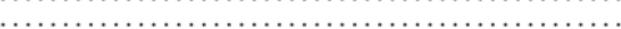

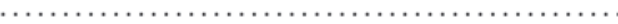

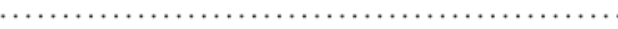

an

ann.

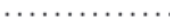

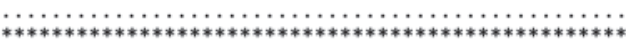

\section{Figure 5}

$\mathrm{V}_{\mathrm{H}}$ gene sequence comparison of IgG1 antibodies (analyzed with IMGT/V-QUEST; see Methods) produced by hybridomas that were derived from the cell fusion of splenocytes with P3U1 plasmacytoma cells. Alignment of $\mathrm{V}_{\mathrm{H}}$ gene sequences in group 1 of Table 3 . CDRs are underlined. Blue, CDR1; red, CDR2; green, CDR3. 
A

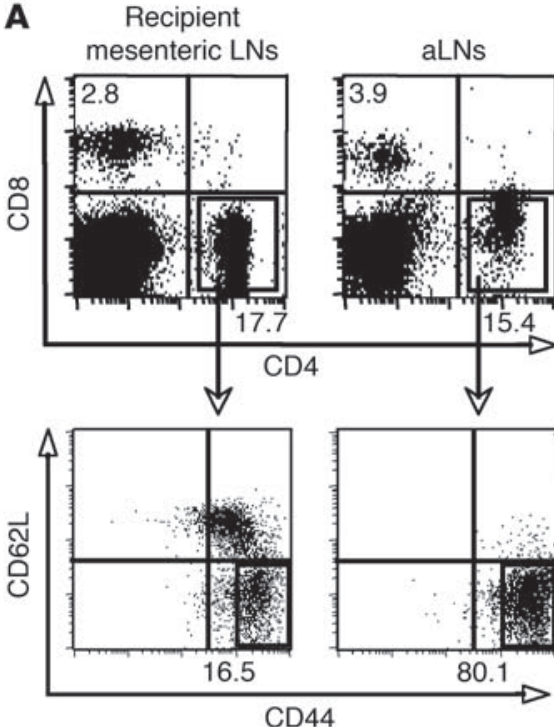

B
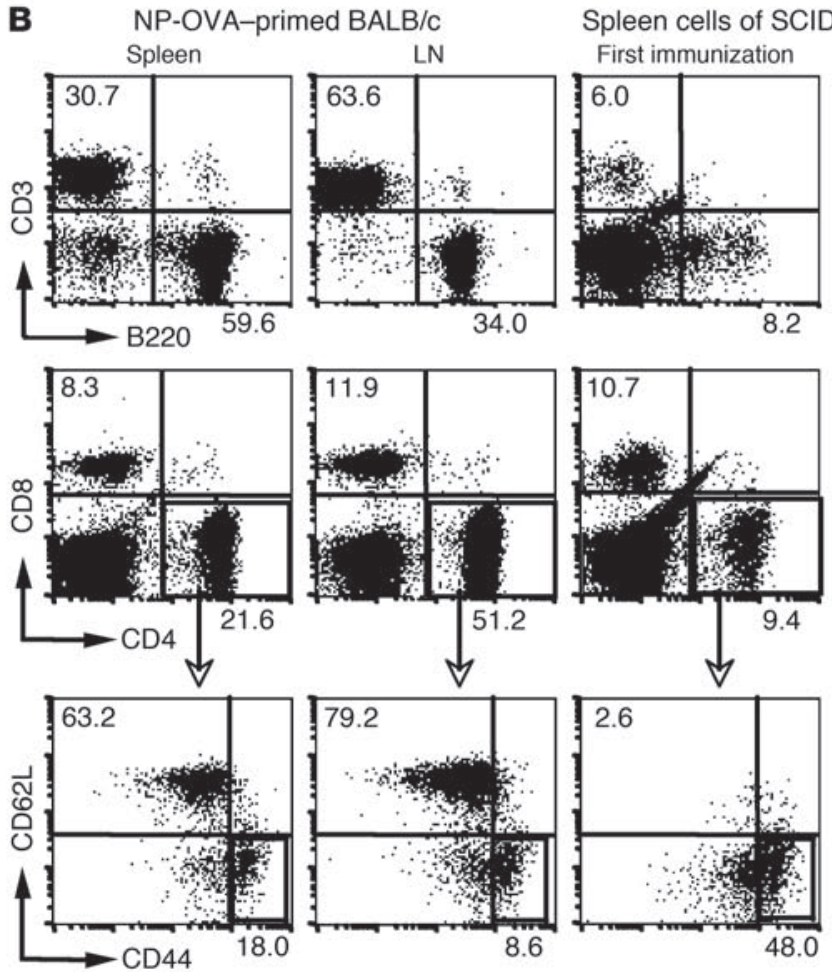

Spleen cells of SCID mice carrying aLNs First immunization Second immunization
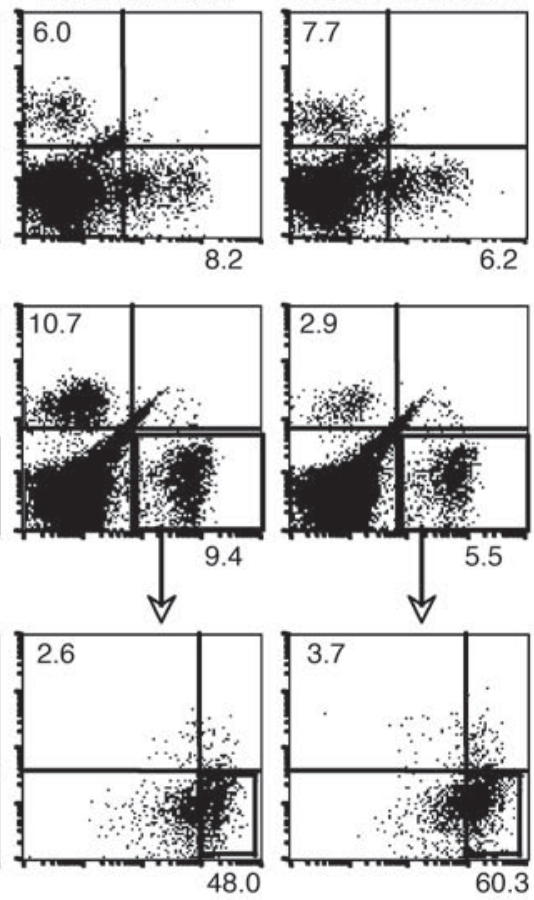

\section{Figure 6}

Enrichment of memory-type (CD44hi CD62 $L^{10}$ ) CD4+ $\mathrm{T}$ cells in aLNs or spleens of aLN-transplanted SCID mice. (A) FACS plots demonstrating an increase in the CD44 ${ }^{\text {hi }} \mathrm{CD}$ 2L $^{\text {lo }}$ memorytype CD4 ${ }^{+} \mathrm{T}$ cell population in aLNs compared with that in lymph nodes of recipient BALB/C mice. Downward pointing arrows indicate the $\mathrm{CD} 44 / \mathrm{CD} 62 \mathrm{~L}$ profile of cells within the $\mathrm{CD} 4^{+} \mathrm{T}$ cell gate. (B) FACS plots demonstrating an increase in the CD44 ${ }^{\text {hiCD62 }}$ lo memory-type CD4+ $T$ cell population in the spleens of aLN-transplanted SCID mice, regardless of antigen stimulation, compared with that of lymph nodes or spleens in normal BALB/c mouse preimmunized with the same doses of NP-OVA. Numbers in the plots in $A$ and $\mathbf{B}$ indicate the percentage of cells within the CD62 ${ }^{\circ} / \mathrm{CD}_{4} 4^{+}$gate. (C) Immunohistochemical staining of $\mathrm{CD} 127^{+} \mathrm{T}$ cells in aLNs. Three weeks after aLNs were formed in NP-OVA preimmunized BALB/c mice, aLNs and lymph nodes of recipient BALB/c mice were stained with FITC-labeled antimouse CD3, anti-mouse CD127 antibodies, and Alexa Fluor 594-conjugated anti-rat IgG. Most $\mathrm{CD}^{+} \mathrm{T}$ cells were also CD127+.

in the lymph nodes of recipient mice. Although the relationship between IL-7 and memorytype $\mathrm{CD} 4^{+} \mathrm{T}$ cells is poorly understood, there are reports that IL-7 contributes to the maintenance of CD4 and CD8 $\mathrm{T}$ cell memory pools $(30,31)$, suggesting that memory $\mathrm{CD} 4^{+} \mathrm{T}$ cells may be enriched in aLNs. (c) The B cell profile in spleens of aLN-transplanted SCID mice after the second immunization showed a high frequency of IgG1 NP-specific AFCs and germinal center $B$ cells as well as an enrichment of memory-type $\mathrm{B}$ cells, consistent with their presence in normal secondary lymphoid tissues $(32,33)$.

Analysis of $\mathrm{V}_{\mathrm{H}}$ gene sequences of IgG1 NPspecific antibodies produced in aLNs and aLNderived spleen cells in SCID mice showed that extensive somatic hypermutation occurred in the single VDJ-rearranged B cell clone, and monoclonal or oligoclonal AFCs secreting a high-affinity antibody were expanded, suggesting the induction of efficient affinity maturation in aLNs.

In support of this notion, large amounts of antigen-specific antibody were found in the serum of aLN-transplanted SCID mice (Table 1). Interestingly, autoantibodies such as antibody to double-stranded DNA were not detected in the sera of aLN-transplanted SCID mice (data not shown), indicating that normal immunoregulatory mechanisms are intact in the context of the aLN. Other features of the aLN system are noteworthy but enigmatic: (a) Memory-type CD4 ${ }^{+}$ $\mathrm{T}$ cells $\left(\mathrm{CD} 44^{+} \mathrm{CD} 62 \mathrm{~L}^{\text {lo }}\right)$ were enriched in aLNs as well as spleens of SCID recipient mice. After transplantation of aLNs into SCID mice, memory-type $\mathrm{CD}^{+} \mathrm{T}$ cells in aLNs migrated to the SCID spleens as a result of immunization, leading to enrichment of these cells in the spleen. (b) $\mathrm{CD}^{+} \mathrm{T}$ cells positive for CD127 (also referred to as IL-7 receptor $\alpha$ ) were also enriched in aLNs, but were barely detectable
Perhaps the most striking feature of our model is the rapid production of very large amounts of antigen-specific isotype-switched antibody upon transplantation of aLNs into SCID mice. In the future, appropriate modifications of the aLNs may be considered as a therapeutic option for immunodeficiency states, as a treatment of refractory infectious diseases, and as a new immunointervention method against cancer.

\section{Methods}

Antibodies and antigens. Fluorescein-, phycoerythrin-, or biotin-labeled or unlabeled anti-B220 (RA3-6B2), anti-CD4 (RM4-5), anti-CD8a (53-6.7), anti-CD44, anti-CD62L, anti-CD3, anti-CD38, anti-CD127, anti-CCR7, anti-CXCR5, anti-FDC-M1, anti-IgG1, and goat anti-hamster IgG as well 

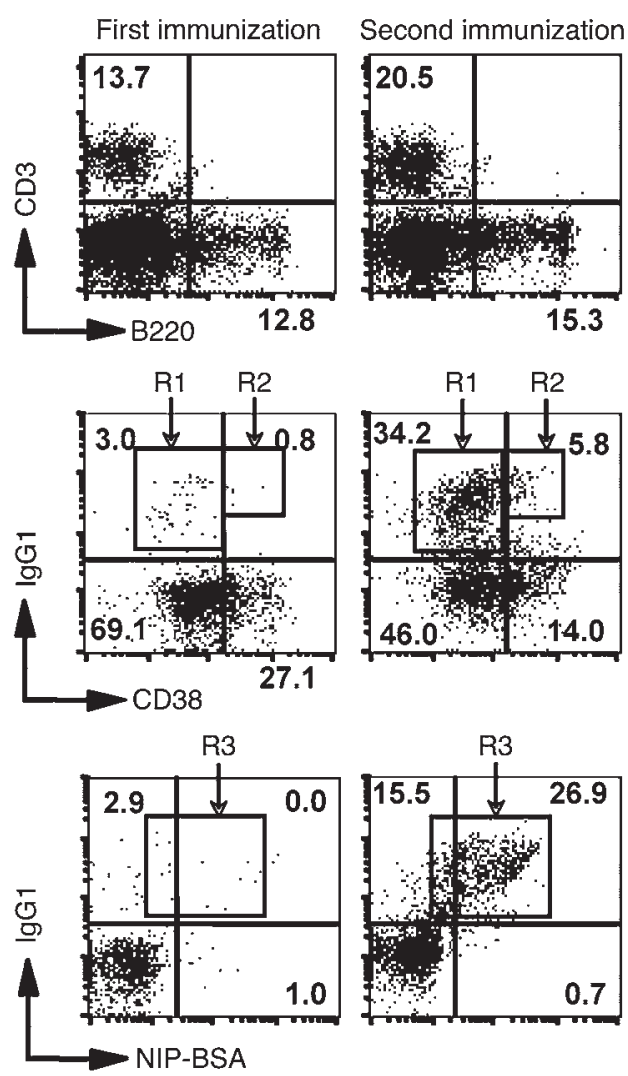

as fluorescein- or phycoerythrin-labeled streptavidin were all purchased from BD Biosciences - Pharmingen. Qdot 605 streptavidin conjugate and Alexa Fluor 594-conjugated anti-rat IgG were purchased from Invitrogen. NP-OVA and NIP-BSA were purchased from Biosearch Technologies. PTX was provided by S. Fagarasan (RIKEN Institute, Yokohama, Japan). FTY720 was a gift from Aventis Pharmaceutical Co.

\section{Figure 7}

$B$ cell profile in spleens of aLN-transplanted SCID mice. FACS plots show germinal center B cells (R1), memory B cells (R2), and IgG1 NPspecific AFCs (R3) after the first and second immunizations. Numbers in the plots are the percentage of cells within the indicated gates.

Mice. BALB/cAnNcrj mice and SCID mice (C.B-17/IcrCrj-scid/scid) were purchased from Charles River Japan Inc. EGFP transgenic mice [C57BL/6Tg14(act-EGFP) Osb Y01] on the BALB/c background were obtained from H. Kiyono (Tokyo University, Tokyo, Japan). All mice were housed under specific pathogen-free conditions in the RCAI animal facility. All experiments using mice described herein were approved by the RCAI animal use committee and were performed in accordance with the applicable guidelines and regulations.

Immunization. For primary immunization, $100 \mu \mathrm{g} \mathrm{NP}{ }_{15}$-OVA precipitated in alum was injected i.p. into 7- to 10 -week-old BALB/c mice. After 4 or more weeks, mice were used as donors for the generation of aLNs. For immunization of naive $\mathrm{BALB} / \mathrm{c}$ or SCID mice that had received transplanted aLNs, $\mathrm{NP}_{15}$-OVA (100 or $10 \mu \mathrm{g}$ ) was injected i.v. at 1 day to 4 weeks after transfer of aLNs.

Stromal cell line. The BALB/c thymus-derived stromal cell line expressing LT $\alpha$, TEL-2-LT $\alpha(9,10)$, was cultured in RPMI 1640 supplemented with $10 \%$ fetal bovine serum.

BM-derived DCs. BM-derived DCs were prepared as previously described (11), with slight modifications. Briefly, BM cells were collected from tibias and femurs of BALB/c mice (7-12 weeks old) by flushing with PBS, after which the cell suspension was filtered through nylon mesh to remove small tissue pieces and debris. BM cells $\left(2 \times 10^{5} / \mathrm{ml}\right)$ were cultured in RPMI 1640 with $10 \%$ fetal calf serum supplemented with $5 \mathrm{ng} / \mathrm{ml}$ recombinant mouse GM-CSF (PeproTech). The medium was changed every 4 days, and nonadherent cells were harvested after 7-9 days of culture. Final maturation of DCs was induced by incubation of the nonadherent cells with LPS (Sigma-Aldrich) at $1 \mu \mathrm{g} / \mathrm{ml}$ for the final 17-24 hours of culture. NP-OVA $(100 \mu \mathrm{g} / \mathrm{ml})$ was added during the final incubation together with LPS to pulse the DCs with antigen. DCs were extensively washed before renal subcapsular transplantation to avoid trace LPS carryover.

\section{Figure 8}

Effect of PTX and FTY720 on the distribution of NP-specific AFCs after aLN transplant into SCID mice. (A-C) Number of NP-specific AFCs in aLNs $(\mathbf{A})$, spleens $(\mathbf{B})$, or BM (C) of recipient SCID mice transplanted with aLNs with or without PTX treatment. (D) Number of NP-specific AFCs in aLNs of SCID mice that received FTY720 administration on days 0,6 , and/or 14 relative to aLN transplantation (day 0). -, without PTX treatment and without NP. OVA stimulation.
A
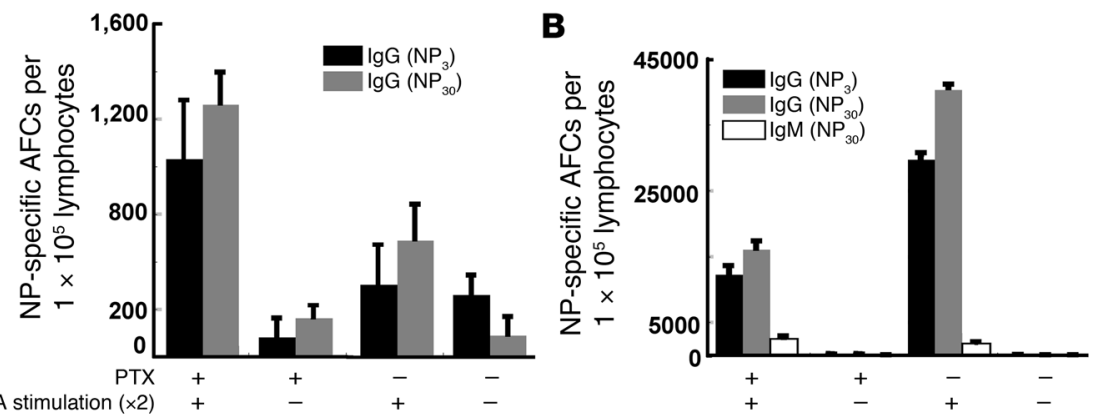

C

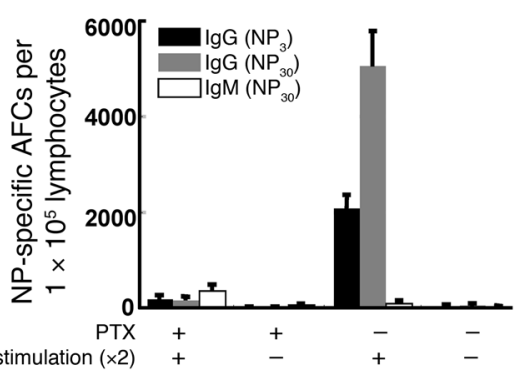

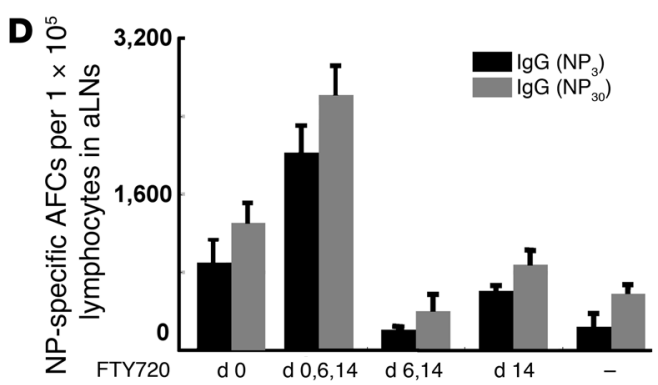


A

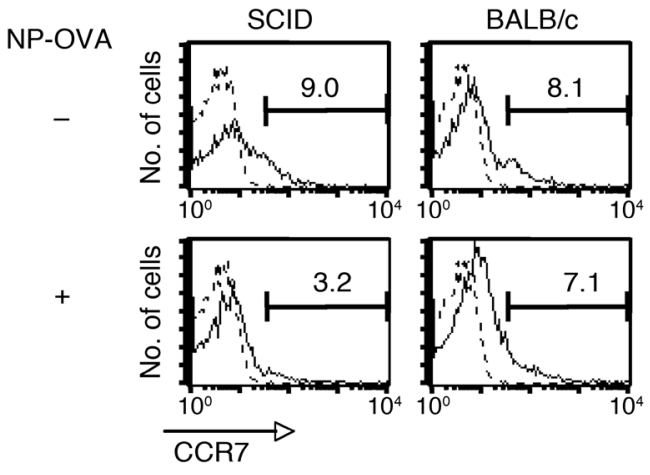

SCID
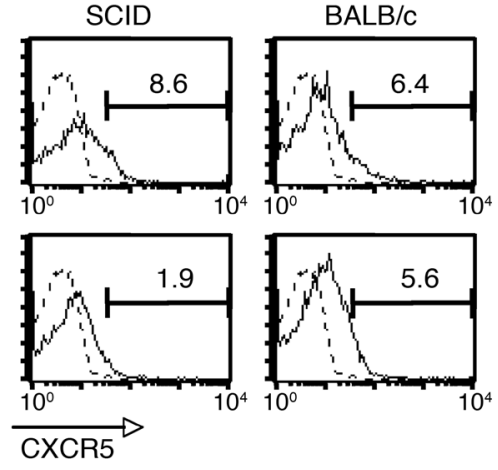

B

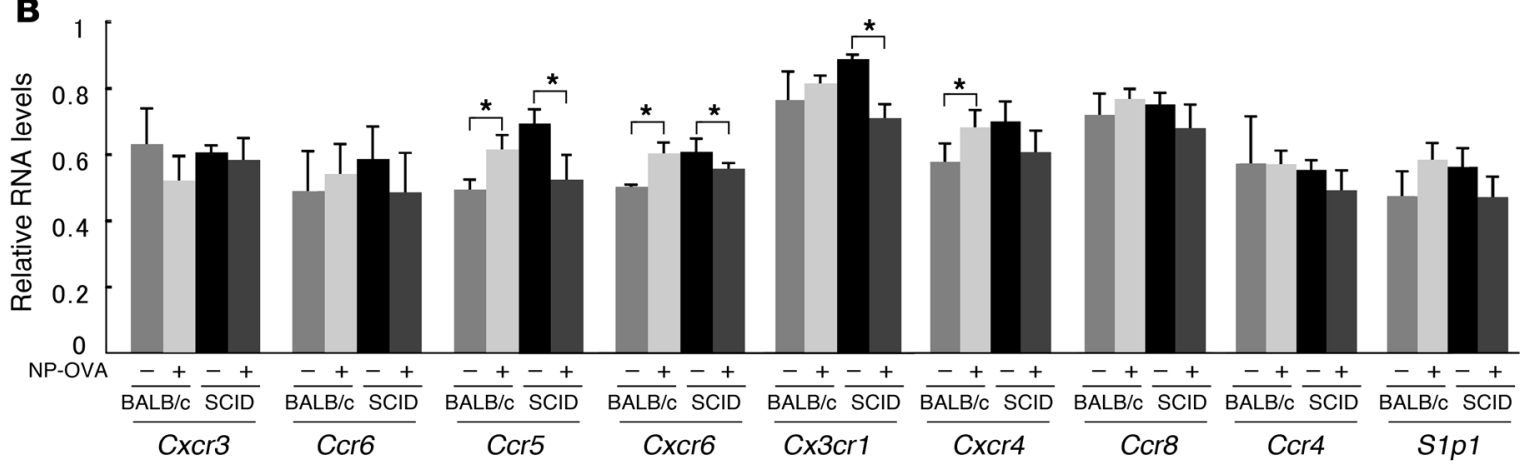

Figure 9

Decreased expression of chemokine receptors on lymphocytes in aLNs (transplanted into SCID mice after the first immunization) by i.v. injection of NP-OVA. (A) CCR7 and CXCR5 expression FACS profiles on lymphocytes in aLNs of recipient SCID or naive BALB/c mice. (B) Changes in chemokine receptor RNA levels as assessed by quantitative RT-PCR in aLNs of recipient SCID or naive BALB/c mice after antigen stimulation. -, no antigen stimulation; +, immunization with NP-OVA (100 $\mu \mathrm{g} / \mathrm{mouse})$. The $y$ axis shows numbers of cells (A) and the relative RNA levels (B). The expression of each receptor RNA level was normalized to the expression of glyceradehyde-3-phosphate dehydrogenase $(g a p d h) .{ }^{*} P<0.1$.

Generation of aLNs. Mixtures of TEL-2-LT $\alpha$ stromal cells and activated DCs were absorbed into a cubic sponge-like collagenous scaffold (Collagen Sponge, CS-35; KOKEN). This matrix is made of insoluble collagen prepared from bovine Achilles tendon by a freeze-dry method and is used primarily for the purpose of in vitro $3 \mathrm{D}$ or high-density cell culture. The shape of the pores of the matrix is not uniform, but the estimated pore size is 50-300 $\mu \mathrm{m}$. The collagen sponge, which has the appearance of 1-mm-thick filter paper, was cut into square pieces of approximately $3 \mathrm{~mm}^{2}$, a size that was kept constant for all experiments. The stromal cells were harvested by trypsin/EDTA and washed once with culture media and twice with PBS. The mature activated BM-derived DCs were extensively washed 2 times with culture media and 3 times with PBS to avoid trace LPS. The cells, a mixture of $1 \times 10^{6}$ stromal cells and $1 \times 10^{6} \mathrm{DCs}$ per sponge, were suspended in $10 \mu \mathrm{l}$ of PBS. The cell suspension was then placed onto a piece of the collagen sponge, which was squeezed several times to absorb cells into the scaffold. The matrix-embedded cells were maintained on ice and kept moist throughout this procedure and immediately implanted into the renal subcapsular spaces of $\mathrm{NP}_{15}$-OVA preimmunized $\mathrm{BALB} / \mathrm{c}$ mice. In most cases, cells were placed at the upper and lower poles of each kidney ( 4 transplants per mouse). Three weeks later, the generated aLNs were removed from the mice, and the mouse sera were collected for further analyses. For transfer experiments, aLNs were harvested from the renal subcapsular space, kept on ice in PBS, and then transplanted into the renal subcapsular spaces of naive or SCID mice. One day after the transplantation, $100 \mu \mathrm{g}$ of $\mathrm{NP}_{15^{-}}$ OVA was administered i.v., and sera were collected 4 days later. The aLNs were also recovered and subjected to immunohistochemical analysis. In the case of aLN transplantation into SCID mice, $100 \mu \mathrm{g}$ of $\mathrm{NP}_{15}$-OVA was administered i.v., and a second i.v. immunization with $10 \mu \mathrm{g}$ of $\mathrm{NP}_{15}-\mathrm{OVA}$ was performed 1 week later. Four days after the second NP-OVA injection, sera were collected and aLNs were harvested.

Immunohistochemical staining. Lymphoid organs and transplanted aLNs were embedded in Tissue-Tek OCT compound (Sakura), and snap frozen in liquid nitrogen. 5 - $\mu \mathrm{m}$-thick cryostat sections were prepared and placed on APS-coated glass slides (Matsunami Glass Ind. Ltd.). Sections were fixed with cold acetone for 5 minutes, dried, and kept at $-80^{\circ} \mathrm{C}$ until use. After blocking with $5 \%$ normal rat serum and $1 \%$ BSA in TBS-T (Tris-buffered saline with $0.005 \%$ Tween 20 ) for 1 hour at $20^{\circ} \mathrm{C}$, sections were incubated for 1 hour at $20^{\circ} \mathrm{C}$ with appropriate antibodies or streptavidin-fluorochrome reagent diluted in the blocking buffer and were washed in PBS at each stage 3 times every 5 minutes.

ELISPOT. The frequency of high- and low-affinity NP-specific AFCs among splenocytes or cells collected from the aLNs was estimated by ELISPOT using $\mathrm{NP}_{3}$-BSA- and $\mathrm{NP}_{30}$-BSA-coated filter plates (for highand low-affinity AFCs, respectively). Hydrophobic PVDF filters on MultiScreenIP Filter Plates (MAIPS4510; Millipore) were coated with $50 \mu \mathrm{g} / \mathrm{ml}$ $\mathrm{NP}_{3}$-BSA, $\mathrm{NP}_{30}$-BSA, or BSA in PBS at $4^{\circ} \mathrm{C}$ overnight, and then blocked with $1 \%$ BSA in PBS. Splenocytes $\left(10^{5}\right.$ cells/well) or cells from transplants $\left(0.2-1 \times 10^{5}\right.$ cells/well) were incubated for 2 hours and washed once with PBS containing $50 \mathrm{mM}$ EDTA, twice with TBS-T, and once with PBS. After washing, filters were visualized with BCIP/NBT (Chemicon International) and AEC (BD Biosciences - Pharmingen).

Measurement of serum NP-specific IgG1 and IgM isotype antibodies. Antibodies specific for the NP-hapten were measured by ELISA. In brief, 96-well assay plates were coated with $50 \mu \mathrm{g} / \mathrm{ml} \mathrm{NP}_{3^{-}}$or $\mathrm{NP}_{30}$-BSA in PBSN (PBS 
containing $0.05 \% \mathrm{NaN}_{3}$ ) at $4{ }^{\circ} \mathrm{C}$ overnight and blocked with $0.5 \% \mathrm{BSA}$ in TBS-T. Serially diluted sera were added to each well and incubated at $4^{\circ} \mathrm{C}$ overnight or at $37^{\circ} \mathrm{C}$ for 1 hour. On each plate, serially diluted sera pooled from primary immunized mice and those boosted once with $\mathrm{NP}_{15}$-OVA were also included as controls. After washing with TBS-T, plates were incubated with HRP-conjugated goat anti-mouse IgG1 at $20^{\circ} \mathrm{C}$ for 2 hours. HRP activity was detected using a TMB Microwell Peroxidase Substrate System (KPL), and optical densities were determined at $450 \mathrm{~nm}$. The concentration of IgG1 NP-specific antibody was estimated by comparison to standard curves created from the pooled IgG1 NP-specific Ig on each plate, used as a positive control.

RT-PCR for amplification of the NP-specific $V_{H}$ gene and sequencing. Total RNA was purified from hybridomas using TRIzOL (Invitrogen). Each cDNA, synthesized from $3 \mu \mathrm{g}$ of total RNA using oligo(dT)-primed reverse transcription, was used as a PCR template. The upstream PCR primer was 5'-AGGTGTCCACTCCCAGGTC-3' (for $\mathrm{V}_{\mathrm{H}} 2$ ), and the downstream primer was 5'-CAGGTCACTGTCACTGGCTC-3' (for mCg1-4). PCR was performed with KOD DNA polymerase (TOYOBO) for 40 cycles of 15 seconds at $94^{\circ} \mathrm{C}$, 30 seconds at $63^{\circ} \mathrm{C}$, and 40 seconds at $68^{\circ} \mathrm{C}$. The PCR products was cloned into PCR 4Blunt-TOPO (Invitrogen) and transformed into TOP 10 bacteria. Resultant colonies were randomly picked, and the $\mathrm{V}_{\mathrm{H}}$ gene sequences were obtained by direct plasmid sequencing using the Big Dye Terminator v3.1 Cycle Sequencing Kit (Applied Biosystems). The $\mathrm{V}_{\mathrm{H}}$ gene sequences were analyzed with IMGT/V-QUEST (http://imgt.cines.fr/textes/vquest/).
Real-time PCR for S1P or chemokine receptor $m R N A$ quantitation. Total RNA was purified from spleen cells of SCID or naive mice using TRIzOL (Invitrogen). Each cDNA, synthesized using High-Capacity cDNA Reverse Transcription Kits (Applied Biosystems), was used as a PCR template. PCR was performed using TaqMan gene expression assays and TaqMan PreAmp Master Mix Kit (Applied Biosystems).

Statistics. All the statistical analyses were performed by using an unpaired 2-tailed Student's $t$ test. A $P$ value of less than 0.1 was considered significant.

\section{Acknowledgments}

We thank Peter Burrows for helpful discussions and assistance with translation. This work was supported by a Grant-in-Aid (16043238) from the Ministry of Education, Culture, Sports, Science, and Technology of Japan.

Received for publication September 18, 2006, and accepted in revised form January 9, 2007.

Address correspondence to: Takeshi Watanabe, Research Unit for Immune Surveillance, Research Center for Allergy and Immunology, RIKEN Yokohama Institute, 1-7-22 Suehiro-cho, Tsurumi, Yokohama 230-0045, Japan. Phone: 81-45-503-7025; Fax: 81-45503-7004; E-mail: wtakeshi@rcai.riken.jp.
1. von Andrian, U.H., and Mempel, T.R. 2003. Homing and cellular traffic in lymph nodes. Nat. Rev. Immunol. 3:867-878.

2. Miyasaka, M., and Tanaka, T. 2004. Lymphocyte trafficking across high endothelial venules: dogmas and enigmas. Nat. Rev. Immunol. 4:360-370.

3. De Togni, P., et al. 1994. Abnormal development of peripheral lymphoid organs in mice deficient in lymphotoxin. Science. 264:703-707.

4. Banks, T.A., et al. 1995. Lymphotoxin-alpha-deficient mice. Effects on secondary lymphoid organ development and humoral immune responsiveness. J. Immunol. 155:1685-1693.

5. Fu, Y.X., and Chaplin, D.D. 1999. Development and maturation of secondary lymphoid tissues. Annu. Rev. Immunol. 17:399-433.

6. Crowe, P.D., et al. 1994. A lymphotoxin-beta-specific receptor. Science. 264:707-710.

7. Force, W.R., et al. 1995. Mouse lymphotoxin-beta receptor. Molecular genetics, ligand binding, and expression. J. Immunol. 155:5280-5288.

8. Shinkura, R., et al. 1999. Alymphoplasia is caused by a point mutation in the mouse gene encoding Nf-kappa b-inducing kinase. Nat. Genet. 22:74-77.

9. Suematsu, S., and Watanabe, T. 2004. Generation of a synthetic lymphoid tissue-like organoid in mice. Nat. Biotechnol. 22:1539-1545.

10. Nakashima, M., et al. 1990. Selective elimination of double-positive immature thymocytes by a thymic epithelial cell line. Eur. J. Immunol. 20:47-53.

11. Sato, K., et al. 2003. Regulatory dendritic cells protect mice from murine acute graft-versus-host disease and leukemia relapse. Immunity. 18:367-379.

12. Chiba, K., et al. 2006. Role of sphingosine 1-phosphate receptor type 1 in lymphocyte egress from secondary lymphoid tissues and thymus. Cell. Mol. Immunol. 3:11-19.

13. Taha, T.A., Argraves, K.M., and Obeid, L.M. 2004.
Sphingosine-1-phosphate receptors: receptor specificity versus functional redundancy. Biochim. Biophys. Acta. 1682:48-55.

14. Sugimoto, N., et al. 2003. Inhibitory and stimulatory regulation of Rac and cell motility by the G12/13-Rho and Gi pathways integrated downstream of a single $G$ protein-coupled sphingosine-1-phosphate receptor isoform. Mol. Cell. Biol. 23:1534-1545.

15. Siehler, S., and Manning, D.R. 2002. Pathways of transduction engaged by sphingosine 1-phosphate through $\mathrm{G}$ protein-coupled receptors. Biochim. Biophys. Acta. 1582:94-99.

16. Anliker, B., and Chun, J. 2004. Lysophospholipid G protein-coupled receptors. J. Biol. Chem. 279:20555-20558.

17. Mandala, S., et al. 2002. Alteration of lymphocyte trafficking by sphingosine-1-phosphate receptor agonists. Science. 296:346-349.

18. Brinkmann, V., and Lynch, K.R. 2002. FTY720: targeting G-protein-coupled receptors for sphingosine 1-phosphate in transplantation and autoimmunity. Curr. Opin. Immunol. 14:569-575.

19. Webster, B., et al. 2006. Regulation of lymph node vascular growth by dendritic cells. J. Exp. Med. 203:1903-1913.

20. Drayton, D.L., et al. 2006. Lymphoid organ development: from ontogeny to neogenesis. Nat. Immunol. 7:344-353.

21. Chen, S.C., et al. 2002. Ectopic expression of the murine chemokines CCL21a and CCL21b induces the formation of lymph node-like structures in pancreas, but not skin, of transgenic mice. J. Immunol. 168:1001-1008.

22. Fan, L., et al. 2000. Cutting edge: ectopic expression of the chemokine TCA4/SLC is sufficient to trigger lymphoid neogenesis. J. Immunol. 164:3955-3959.

23. Drayton, D.L., et al. 2003. Ectopic LT alpha beta directs lymphoid organ neogenesis with concomitant expression of peripheral node addressin and a HEV-restricted sulfotransferase. J. Exp. Med. 197:1153-1163.

24. Kratz, A., et al. 1996. Chronic inflammation caused by lymphotoxin is lymphoid neogenesis. J. Exp. Med. 183:1461-1472.

25. Luther, S.A., et al. 2000. BLC expression in pancreatic islets causes B cell recruitment and lymphotoxin-dependent lymphoid neogenesis. Immunity. 12:471-481.

26. Luther, S.A., et al. 2002. Differing activities of homeostatic chemokines CCL19, CCL21, and CXCL12 in lymphocyte and dendritic cell recruitment and lymphoid neogenesis. J. Immunol. 169:424-433.

27. Aloisi, F., and Pujol-Borrell, R. 2006. Lymphoid neogenesis in chronic inflammatory diseases. Nat. Rev. Immunol. 6:205-217.

28. Rosen, H., and Goetzl, E.J. 2005. Sphingosine 1-phosphate and its receptors: an autocrine and paracrine network. Nat. Rev. Immunol. 5:560-570.

29. Hla, T. 2003. Signaling and biological actions of sphingosine 1-phosphate. Pharmacol. Res. 47:401-407.

30. Bradley, L.M., Haynes, L., and Swain, S.L. 2005. IL-7: maintaining T-cell memory and achieving homeostasis. Trends Immunol. 26:172-176.

31. Fukuyama, S., et al. 2002. Initiation of NALT organogenesis is independent of the IL-7R, LTbetaR, and NIK signaling pathways but requires the Id 2 gene and CD3(-)CD4(+)CD45(+) cells. Immunity. 17:31-40.

32. Ridderstad, A., and Tarlinton, D.M. 1998. Kinetics of establishing the memory B cell population as revealed by CD38 expression. J. Immunol. 160:4688-4695.

33. McHeyzer-Williams, L.J., and McHeyzer-Williams, M.G. 2005. Antigen-specific memory B cell development. Annu. Rev. Immunol. 23:487-513. 